\title{
Dimensiones del Desarrollo Endógeno en la Gerencia de las Universidades*
}

\section{Dimensions of endogenous development in universities management}

\author{
Sugey Martha Issa Fontalvo* \\ Sena Regional Magdalena, Santa Marta -Colombia \\ Recibido: Febrero 19 de 2017 \\ Aceptado: Junio 07 de 2017 \\ Aceptado: Julio 02 de 2017
}

\section{Resumen}

El presente artículo es producto de la investigación cuyo propósito fue caracterizar las dimensiones del desarrollo endógeno en la gerencia de las universidades del distrito de Santa Marta. La metodología utilizada fue bajo el paradigma de investigación positivista, no experimental, transeccional, descriptiva, de campo. La población finita estuvo constituida por 37 empleados. Para la recolección de datos se elaboró un instrumento utilizando las categorías de respuestas tipo Likert, validado por siete expertos en el área. La confiabilidad del instrumento fue determinada por el método Alfa de Cronbach presentando 0.96 de confiabilidad. Los resultados de esta investigación indican que, la dimensión Social, Ética, Cultural, Tecnológica, Organizacional y Política están considerablemente presentes en la gerencia de las universidades, mientras que la dimensión Económica tiene un bajo porcentaje sobre la presencia de sus características. Se concluye que, la gerencia de las universidades en estudio, considera las costumbres que se engendran en el seno de una sociedad, además, que la tecnología es clave para los procesos productivos, estimula los valores morales, ayuda a la generación de servicios para la región y propone pautas para consolidar la estrategia de desarrollo endógeno.

Palabras clave: Desarrollo endógeno, gerencia innovadora, gerencia universitaria.

\begin{abstract}
This research paper aims to characterize the dimensions of endogenous development in university management in Santa Marta. A positivist research paradigm, non experimental, transactional, descriptive and field was used as methodology. 37 employees were sampled. For data collection, responses categories in the Likert scale was used as instrument validated by seven experts in this area. The instrument reliability was determined by Cronbach's alpha method with 0.96 reliability. As a result, Social, ethics, cultural, technological, organizational and political dimensions are part of universities management under consideration, in contrast with the economic dimension characteristics which has a low percentage. As a conclusion in this research, the universities management in Santa Marta, considers customs are spawned in a society, as well as technology is key for productive processes, stimulates moral values, helps services generation for the local region and proposes guidelines to consolidate the endogenous development strategy.
\end{abstract}

Keywords: Endogenous development, innovative management, university management.

\section{Este artículo se puede referenciar}

Issa-Fontalvo, S. (2017). Dimensiones del Desarrollo Endógeno en la Gerencia de las Universidades. En Desarrollo Gerencial Revista de la Facultad de Ciencias Económicas Administrativas y Contables de la Universidad Simón Bolívar-Colombia, 9(2), 174-188.

* Este artículo es producto de la investigación "Cultura de Innovación en la Gerencia de las Universidades del distrito de Santa Marta, para el desarrollo endógeno de la región".

${ }^{*}$ Centro de Logística y Promoción Ecoturística del Sena Regional Magdalena. Email: sugeyissa@ hotmail.com. 


\section{1.- Introducción}

América Latina se enfrenta a la necesidad de crear una percepción distinta acerca del papel de la ciencia y la tecnología como instrumento para alcanzar el desarrollo, combatir la pobreza y construir sociedades más equitativas. Asimismo, las diferentes estrategias nacionales deben estar orientadas, por una parte, a la consolidación de capacidades básicas de Investigación, desarrollo e innovación, formación de recursos humanos y generación de entornos favorables para la difusión de la ciencia y la tecnología a escala social para promover el desarrollo endógeno.

El surgimiento del desarrollo endógeno se produce como consecuencia del nuevo paradigma de la globalización, que indujo el incremento de la competencia de los mercados y, consecuentemente, un reordenamiento de los sistemas productivos, pero también de la organización del sistema de ciudades y regiones, para permitir una mayor competitividad nacional, regional y local en el contexto de una economía mundial.

Cabe destacar que el desarrollo endógeno es en sociedad, por tal razón en las organizaciones los procesos de desarrollo están condicionados por factores sociales, la gerencia debe propiciar los factores sociales como el espíritu de trabajo, la capacidad y las reglas que regulan las relaciones entre las personas, premiar el esfuerzo y la ética de trabajo y la capacidad emprendedora que es un valor social que debe ser reconocido dentro de las organizaciones de un territorio.

De acuerdo con Mas (2008), al vincular al Desarrollo Endógeno a la educación lo hace como estrategia de carácter universal, asumiendo abordar el hecho educativo desde una óptica multidimensional, considerando sus aristas sociales, éticas, ecológicas, culturales, políticas, económicas, tecnológicas, unidas estas a fin de mejorar la calidad de vida de manera conjunta. Esto significa que un gerente con un principio de orientación al bien común, modera la competitividad y busca que dentro de la organización todos ganen, se preocupa por mantener una mentalidad global en función de todo el equipo de la organización, contribuyendo no solo con cada uno de los empleados, sino luchando por todo aquello que es bueno para toda la organización.

Este artículo representa la investigación que se lleva a cabo con la finalidad de caracterizar las dimensiones del desarrollo endógeno en la gerencia de las universidades del distrito de Santa Marta; para esto se plantea en siguiente interrogante: ¿Qué características de las dimensiones del desarrollo endógeno están presentes en la gerencia de las universidades del distrito de Santa Marta?

En este sentido, en principio se describe la fundamentación teórica, la orientación metodológica para la investigación constituida por el enfoque metodológico, la orientación en la tipología de la investigación y la validez y la confiabilidad del instrumento de recolección de la información; y finalmente se presentan 
los resultados de la investigación, las conclusiones y las referencias bibliográficas sobre las que se apoya el artículo sobre la investigación.

\section{2.- Fundamento Teórico}

Mas (2006), considera que en cada dimensión existen elementos diversos y además, estos elementos no se relacionan siempre con la misma regularidad ni en la misma proporción, pues, su desenvolvimiento es cambiante y en última instancia dependen de la imagen objetivo deseada y de la capacidad endogenizadora que le sea propia, se requiere articular, para cada dimensión del desarrollo endógeno, una estrategia singular que a su vez, participe en todo momento, de la Estrategia Maestra.

Este conjunto de acciones o actividades seleccionadas e impulsadas por la totalidad de los miembros de una comunidad para alcanzar el futuro, entendiendo por comunidad una familia, un municipio, una ciudad, una región, un país, una nación o un grupo de países, señala la autora, señalando como dimensiones del desarrollo endógeno, las siguientes:

\section{1.- Dimensión Social}

En un sentido general, Mas (2005) afirma que el desarrollo endógeno es en sociedad, sus logros y propósitos, así como los recursos con los que cuenta, tienen por escenario y teología lo social. En un sentido particular, los procesos de desarrollo están condicionados por factores sociales como el espíritu de trabajo, la capacidad y las reglas que regulan las relaciones entre las personas y las organizaciones del territorio. Así, cuando en una organización se premia el esfuerzo y la ética de trabajo, la capacidad emprendedora es un valor social reconocido, y la movilidad social se estimula, la población está en capacidad de responder a los retos y desafíos, de manera creativa, considera Vásquez (2007).

En el caso del contexto académico, a criterio de Garizabal, Londoño y Uribe (2014), es conveniente que las universidades se basen en una dimensión social pues les permite planear, evaluar, hacer un diagnóstico de la realidad y medir el alcance de su labor social, así como la creación de parámetros que sirvan como base comparativa de su desempeño, capaz de responder a las necesidades e intereses reales de la sociedad

\section{2.- Dimensión Ética}

Todos los actos humanos requieren una valoración previa, según Mas (2005), esta valoración se realiza en atención a unos valores, los cuales, constituyen la esfera moral y cómo esta afecta poderosamente las elecciones y decisiones de las personas ejercen una gran influencia en todos los campos de la vida humana. La ética afecta las valoraciones en cuánto a qué y cómo producir, los procesos empresariales y en última instancia, define un tipo de convivencia en sociedad. De esta forma, la moral se desempeña trascendentalmente en el desarrollo endógeno, pues determina el marco valorativo socialindividual de donde se propone el futuro y se preparan las condiciones necesarias para su consecución 
definitiva.

De otra parte, la visión de la universidad como agente de formación ciudadana responsable es el paradigma que persiguen las acciones éticas. En este sentido, Durán et al. (2016) y Paz et all (2017) señalan que las instituciones de educación, como las universidades, en la búsqueda de propiciar la creación de una cultura social y comunitaria con un basamento ético, han direccionado sus procesos curriculares a promover programas y procesos de desarrollo y apoyo a la sociedad.

Según Botello (2013), plantear la enseñanza universitaria como mucho más que la adquisición de contenidos conceptuales o habilidades técnicas es un reto para docentes que entienden que el compromiso para con la sociedad va más allá, alcanzando también cotas de formación humana y ciudadana. Por eso, son competencias éticas la búsqueda del bien común por encima del personal, la apuesta por el diálogo como modo de construir la verdad colectiva o la sensibilización de los estudiantes hacia la función social de las profesiones.

\section{3.- Dimensión Cultural}

Todo cuanto el ser humano aporta así mismo se expone en la trama cultural, el comportamiento humano se constituye en una de las palancas fundamentales que favorece o frena las estrategias y finalidades del desarrollo endógeno. Desde la perspectiva de la gestión, el elemento común en todas las dimensiones es el ser humano. No existe ética sino se comienza por vivir en sociedad. Según Mas (2005), la cultura es asumida como la forma de vida de las personas, y que cada comunidad, país o nación, presenta una tipología de costumbres propias y singulares, así como creencias y tradiciones, formas de vida, de interacción y de acción, que le otorgan especial particularidad a un conjunto humano y social, siendo la cultura unos de los elementos claves del ámbito de sustentabilidad para el desarrollo endógeno. No es posible adelantar ninguna aproximación al desarrollo endógeno sino se consideran las tradiciones, las costumbres, ritos, creencias y los valores que se engendran en el seno de una sociedad.

En las organizaciones, Davis y Newstrom (2011) definen la cultura como el conjunto de supuestos, creencias, valores y normas que comparten los miembros de una organización. Es factible que la hayan creado conscientemente sus miembros clave o que tan solo ha evolucionado con el paso del tiempo. Constituye un elemento clave del ambiente laboral donde los empleados realizan su trabajo. La idea de la cultura organizacional es hasta cierto punto intangible, ya que no se puede ver ni tocar, si bien está presente y tiene efectos amplios. Del mismo modo, Gibson (2006), explica que la cultura organizacional se compone de los valores, creencias, supuestos, percepciones, normas y patrones de comportamiento comunes a todos los que trabajan en ella. Asimismo, la cultura de la organización es una manera de observar y considerar como funciona el comportamiento en las organizaciones, una perspectiva que se puede adoptar para comprender lo que ocurre. 
Asimismo, Kreitner y Kinicki (2003) señalan que la cultura organizacional como el conjunto de supuestos compartidos e implícitos, que se dan por sentados, en un grupo, el cual determina la manera en que el grupo percibe sus diversos entornos, piensa respecto de ellos y reacciona a ellos mismos.

Tomando como base, las definiciones de los autores mencionados, es importante mencionar que entre ellos hay consenso al considerar que la cultura organizacional es un patrón de valores, creencias y normas que comparten los empleados de una organización y que permite el cumplimiento de la misión, visión y acciones estratégicas de la misma. En este punto se enfocó el concepto de Davis y Newstrom (2011), ya que además de indicar que la cultura es un patrón de valores y creencias, también se considera que influye de manera intangible en el entorno de la organización haciendo que este evolucione a través del tiempo.

Tanto en las organizaciones como en la sociedad, se desarrolla la búsqueda de conocimientos científicos, técnicos y sedan los pasos para el encuentro con la tecnología. Dada una cultura las personas exigen y producen medios económicos y se crean empresas y organizaciones para estos fines. La cultura es abarcarte y envolvente. Cada organización social desde la familia hasta la gran empresa global desarrolla una cultura que participa a su vez de la totalidad. De esta forma, será necesario poseer algunas aproximaciones de la cultura y del desarrollo endógeno.

\section{4.- Dimensión Tecnológica}

Para Morán (2007), la tecnología es una creación humana para obtener medios a menor costo y lograr satisfacer fines, sin embargo, su utilización ha dado origen a grandes conflictos de orden social, ético, ecológico y en general, humanos. La tecnología es una palanca clave para el desempeño de los procesos productivos, para el ejercicio organizacional y para la economía en general.

La tecnología, señala Mas (2005), es uno de los factores críticos para la sustentación y sostenibilidad en la construcción de la visión y la estrategia del Desarrollo Endógeno. Sin embargo, la sociedad actual, con el conjunto de adjetivos calificativos: sociedad de la información, en redes, de la gratuidad, del ocio, globalizada, posmoderna, mantiene como rasgo común la tendencia, cada vez mayor, a crear, utilizar y depender de la tecnología. Podría sostenerse, más bien, que de mantenerse esta predisposición, la sociedad del futuro será el resultado de los alcances en materia de tecnología y no en la realización integral humana.

Alburquerque (2004) afirma que la dimensión tecnológica del desarrollo endógeno implica mantener y dinamizar el potencial de creatividad, innovación y solución de los problemas, para lo cual se requiere un ambiente propicio a la expresión y ampliación de los talentos personales. Satisfacer las necesidades y exigencias de los cambios incesantes en la demanda.

Vázquez (2007) afirma que algunos factores que influyen para aumentar la capacidad de respuesta o de reestructuración productiva son la tasa de innovación regional, la cualificación de los recursos humanos, la 
capacidad tecnológica y emprendedora de las empresas, la flexibilidad de las organizaciones empresariales e instituciones y la integración de empresas, ciudades y regiones en redes competitivas e innovadoras.

\section{5.- Dimensión Económica}

En la economía se presenta la alternativa para la selección, producción y consumo de medios que otorgan satisfacción a los deseos humanos. Con base en las infinitas interacciones sociales y sobre la selección de estos medios se fundamenta la acción económica.

Mas (2005) afirma que el desarrollo endógeno se sustenta sobre un conjunto de relaciones de producción, transformación, distribución y consumo de medios materiales y económicos que se obtienen a partir de la acción de las personas. Esta ocurrencia sella el fenómeno del desarrollo en el ámbito económico. Desde aquí y atendiendo a los constructos de la teoría económica es probable determinar los elementos y relaciones existentes para la red de economía real y nominal.

Asimismo el mismo autor establece que, la red de economía real se concibe como el conjunto de bienes y servicios que se producen en la economía bajo unas condiciones de producción dadas, a lo largo de un periodo de tiempo. La red de economía nominal se refiere a la actividad monetaria y financiera que existe como fundamento y reflejo de la red de producción real. Ambas redes, actuando conjuntamente, dan sustento a la trama económica.

En palabras de Vásquez (2007), la dimensión económica está referida a la capacidad que demuestran las empresas endógenas para organizar los factores productivos con niveles de productividad suficientes para ser competitivos en los mercados, y caracterizada por: una elevada división del trabajo entre las empresas del sistema productivo local, una marcada especialización productiva, una multiplicidad de sujetos económico locales, una fácil difusión de información técnica y comercial mutua, un sistema de formación profesional construido por la propia localidad y, una fuerte integración entre las instituciones y la economía local.

\section{6.- Dimensión Organizacional y Gerencial}

En palabras de Mas (2005), el fenómeno del desarrollo endógeno asigna o impone como elemento clave de sustentación, la existencia de organizaciones eficientes que se dediquen a la producción de bienes y servicios. Además, se requiere la inclusión de otras organizaciones a los fines, de dar apalancamiento al sistema productivo general y a la comunidad social interesada en el desarrollo endógeno.

\section{7.- Dimensión Politica}

En la dimensión política del desarrollo endógeno, Mas (2005), refiere que se pretende trazar los roles, concepciones, y estilos de comportamientos de los entes gubernamentales para apoyar y consolidar la 
estrategia de desarrollo endógeno. Esto significa que la política institucional de participación, representada por las forma de organización un conjunto de políticas territoriales permiten la creación de un entorno económico local favorable, capaz de desplegar las potencialidades locales y de proteger al territorio de interferencias externas, señala Vásquez (2007).

Del mismo modo, Zevallos (2001) advierte que no se debe confundir el desarrollo endógeno de los países en desarrollo con el crecimiento económico equilibrado de las economías capitalistas desarrolladas. El confundir ambos términos desvía la atención de los factores que propician el desarrollo, para centrarse en las condiciones que permiten el crecimiento sin amplias fluctuaciones de la actividad económica y de la ocupación.

\section{3.- Método}

El paradigma epistemológico presente en el estudio fue el positivismo, pues la investigación partió desde una dimensión hipotética hasta llegar a la creación del conocimiento sobre el comportamiento de la variable desarrollo endógeno. Asimismo, esta investigación fue de tipo descriptiva ya que se identificaron las características y el comportamiento de las variables en estudio reflejadas en instituciones de educación superior.

\section{Diseño}

El estudio tuvo un diseño de campo, debido a que se elaboró una encuesta para recolectar la información sobre la realidad de la población estudiada; transeccional, dado que se obtuvieron datos actuales sobre la variable objeto de estudio a través de un instrumento de recolección de datos en una sola oportunidad y en un mismo tiempo; y no experimental, pues en ella no se construyó ninguna situación sobre la variable en estudio, sino que se observaron situaciones ya existentes, no provocadas intencionalmente por el investigador.

\section{Participantes}

La población finita estuvo constituida por 37 empleados vinculados en 5 universidades del distrito de Santa Marta, que conformaron el universo poblacional. En ese orden de ideas, es importante mencionar, que la población en cada organización estuvo constituida por empleados ordinarios, ambos sexos, diferentes edades, ocupando cargos de decanos y jefes de los centros de investigación; en este sentido, se debe señalar que por el reducido número de unidades de observación no se realizó cálculo alguno. Por ello, se considera la técnica muestral de tipo censal, la que Tamayo y Tamayo (2004) define como un censo poblacional describiéndola como aquella donde todos los elementos de la población son incluidos en el estudio. 


\section{Instrumentos}

Como instrumento para la recolección de datos se elaboró un cuestionario utilizando las categorías de respuestas de tipo Likert de 21 ítems, validado por siete expertos en el área. Las categorías de respuesta que contiene el instrumento de recolección de datos son: (5) Muy de acuerdo, (4) De acuerdo, (3) Ni de acuerdo, ni en desacuerdo, (2) En desacuerdo y (1) Muy en desacuerdo.

La confiabilidad del instrumento fue determinada por el método Alfa de Cronbach presentando un 0.96 de confiabilidad y se sustenta sobre la aplicación de pruebas piloto. Por lo tanto, la prueba piloto se realizó seleccionando diez (10) personas distintas a la población en estudio pero con las mismas características abordadas en la investigación. A esta población se le aplicó el mismo instrumento sujeto al estudio, permitiendo recolectar la data en matrices de dos entradas, donde se agruparon las sumatorias de los reactivos, el puntaje total de cada sujeto, la media aritmética de cada una de esas sumatorias y las correspondientes desviaciones estándar, al igual que los diez (10) sujetos seleccionados en el estudio para la ejecución de la prueba en cuestión.

Posteriormente, fue aplicado a la población del estudio constituida por las universidades mencionadas en la muestra. Finalmente, se agruparon los datos haciendo uso de la estadística descriptiva (spss) construyendo las tablas de frecuencia, y posterior análisis e interpretación de los datos obtenidos.

\section{Procedimientos}

Con la finalidad de cumplir con los objetivos establecidos en esta investigación, metódicamente se llevaron a cabo ciertas responsabilidades. Inicialmente, se seleccionó la problemática a estudiar, una problemática que se manifiesta en las necesidades del entorno, orientando el problema hacia la búsqueda de un objetivo, que en este caso es, determinar la cultura de innovación para promover el desarrollo endógeno de la región en la gerencia de las universidades del distrito de Santa Marta. Seguidamente, se procedió al establecimiento, selección y análisis de las diferentes bibliografías existentes con base en la presente investigación, así como al establecimiento de las variables a estudiar.

Luego, se determinó el tipo de investigación, el diseño de la misma y la población y muestra; así como la realización del mapa de variables, especificando las dimensiones e indicadores. Posteriormente, se adecuaron los instrumentos de medición de acuerdo a los objetivos planteados en la presente investigación, y seguidamente, se realizó la aplicación del instrumento a la población que en este caso estuvo conformada por las cinco (5) instituciones de educación superior que existen en el distrito de Santa Marta en Colombia.

Después de la aplicación del instrumento, se seleccionaron las técnicas de tabulación y datos estadísticos, considerando el objetivo del estudio, y a continuación, se realizaron los gráficos 
representativos de los resultados obtenidos. Una vez obtenidos los datos, los resultados fueron analizados para determinar situaciones que dieran respuesta a los indicadores y que permitían cumplir con el objetivo trazado en esta investigación.

\section{4.- Resultados}

Al caracterizar las dimensiones del desarrollo endógeno en la gerencia de las universidades del distrito de Santa Marta, se tuvieron en cuenta las dimensiones Social, Ética, Cultural, Tecnológica, Económica, Organizacional y Política.

En función de los resultados reflejados en la tabla 1 se debe notar que, sobre las características de la dimensión social del desarrollo endógeno, 9 de los sujetos que representan el 25\% de los encuestados ante las afirmaciones contenidas en el cuestionario sobre la dimensión social del desarrollo endógeno respondieron a la categoría de respuesta positiva "Muy de acuerdo", 9 de los sujetos que representan el $24 \%$ seleccionaron la categoría de respuesta "De acuerdo", 9 de los sujetos que representan el 23\% seleccionaron la categoría de respuesta negativa "En desacuerdo", 6 de los sujetos que representan el 17\% de los encuestados seleccionaron la categoría de respuesta "Muy en desacuerdo", y 4 de los sujetos que representan el $10 \%$ de los encuestados seleccionaron la categoría de respuesta "Ni de acuerdo, ni en desacuerdo".

Tabla 1: Dimensiones del desarrollo endógeno

\begin{tabular}{|c|c|c|c|c|c|c|c|c|c|c|c|c|c|c|c|c|}
\hline \multirow{2}{*}{$\begin{array}{c}\text { CATEGORIA } \\
\text { DE RESPUESTA }\end{array}$} & \multicolumn{2}{|c|}{ Social } & \multicolumn{2}{|c|}{ Ética } & \multicolumn{2}{|c|}{ Cultural } & \multicolumn{2}{|c|}{ Tecnol. } & \multicolumn{2}{|c|}{ Económ } & \multicolumn{2}{|c|}{ Organiz } & \multicolumn{2}{|c|}{ Política } & \multirow{2}{*}{$\mathbf{X}$} & \multirow{2}{*}{$\%$} \\
\hline & $\mathrm{Fi}$ & $\%$ & $\mathrm{Fi}$ & $\%$ & $\mathrm{Fi}$ & $\%$ & $\mathrm{Fi}$ & $\%$ & $\mathrm{Fi}$ & $\%$ & $\mathrm{Fi}$ & $\%$ & $\mathrm{Fi}$ & $\%$ & & \\
\hline Muy de acuerdo & 9 & 25 & 9 & 24 & 13 & 35 & 11 & 30 & 5 & 13 & 13 & 35 & 11 & 30 & 10 & 27 \\
\hline De acuerdo & 9 & 24 & 11 & 30 & 4 & 38 & 13 & 34 & 5 & 14 & 2 & 32 & 14 & 37 & 1 & 30 \\
\hline $\begin{array}{l}\text { Ni de acuerdo, ni } \\
\text { en desacuerdo }\end{array}$ & 4 & 10 & 1 & 3 & 2 & 5 & 1 & 3 & 3 & 8 & 2 & 5 & 3 & 7 & 2 & 6 \\
\hline En desacuerdo & 9 & 23 & 8 & 23 & 4 & 10 & 7 & 18 & 13 & 36 & 7 & 18 & 6 & 15 & 8 & 20 \\
\hline $\begin{array}{l}\text { Muy en } \\
\text { desacuerdo }\end{array}$ & 6 & 17 & 8 & 21 & 4 & 12 & 6 & 15 & 11 & 29 & 4 & 10 & 4 & 11 & 6 & 10 \\
\hline TOTAL & 37 & 100 & 37 & 100 & 37 & 100 & 37 & 100 & 37 & 100 & 37 & 100 & 37 & 100 & 37 & 100 \\
\hline
\end{tabular}

Fuente: Elaboración propia (2016).

Con respecto a la dimensión ética se observa que, 11 de los sujetos que representan el $30 \%$ de los encuestados seleccionaron la categoría de respuesta positiva "De acuerdo", 9 de los sujetos que representan el 24\% de los encuestados seleccionaron la categoría de respuesta "Muy de acuerdo", 8 de los sujetos que representan el $23 \%$ de los encuestados seleccionaron la categoría de respuesta "En desacuerdo", 8 de los sujetos que representan el 21\% de los encuestados seleccionaron la categoría de respuesta "Muy de acuerdo", y 1 de los sujetos que representan el 3\% de los encuestados selecciono la categoría de respuesta "Ni de acuerdo, ni en desacuerdo".

En cuanto a la dimensión cultural, los resultados revelan que 14 de los sujetos que representan el 38\% 
de los encuestados seleccionaron la categoría de respuesta "De acuerdo", 13 de los sujetos que representan el 35\% de los encuestados seleccionaron la categoría de respuesta "Muy de acuerdo", 4 de los sujetos que representan el 12\% de los encuestados seleccionaron la categoría de respuesta "Muy de acuerdo", 4 de los sujetos que representan el $10 \%$ de los encuestados seleccionaron la categoría de respuesta "En desacuerdo", y 2 de los sujetos que representan el 5\% de los encuestados seleccionaron la categoría de respuesta "Ni de acuerdo, ni en desacuerdo".

En la dimensión tecnológica se puede observar que, los resultados arrojaron que 13 de los sujetos que representan el 34\% de los encuestados seleccionaron la categoría de respuesta positiva "De acuerdo", 11 de los sujetos que representan el 30\% de los encuestados seleccionaron la categoría de respuesta "Muy de acuerdo", 7 de los sujetos que representan el 18\% de los encuestados seleccionaron la categoría de respuesta negativa "En desacuerdo", 6 sujetos que representan el 15\% de los encuestados seleccionaron la categoría de respuesta "Muy en desacuerdo", solo 1 de los sujetos que representa el 3\% de los encuestados seleccionaron la categoría de respuesta "Ni de acuerdo, ni en desacuerdo".

Sobre la dimensión económica, los resultados arrojaron que 13 de los sujetos que representan el 36\% de los encuestados respondieron en forma negativa seleccionando la categoría de respuesta "En desacuerdo", 11 de los sujetos que representan el 29\% de los encuestados seleccionaron la categoría de respuesta "Muy en desacuerdo", 5 sujetos que representan el 14\% de los encuestados seleccionaron la categoría de respuesta positiva "De acuerdo", 5 sujetos que representan el 13\% de los encuestados seleccionaron la categoría de respuesta "Muy de acuerdo", y 3 de los sujetos que representan el 8\% de los encuestados mantienen una posición neutral al seleccionar la categoría de respuesta "Ni de acuerdo, ni en desacuerdo".

En cuanto a la dimensión organizacional y gerencial los resultados arrojaron que, 13 de los sujetos que representan el 35\% de los encuestados seleccionaron la categoría de respuesta positiva "Muy de acuerdo", 12 de los sujetos que representan el 32\% de los encuestados seleccionaron la categoría de respuesta "De acuerdo", 7 sujetos que representan el $18 \%$ de los encuestados seleccionaron la categoría de respuesta negativa "En desacuerdo", 4 de los sujetos que representan el 10\% de los encuestados seleccionaron la categoría de respuesta "Muy en desacuerdo", y 2 de los sujetos que representan el 5\% de los encuestados seleccionaron la categoría de respuesta "Ni de acuerdo, ni en desacuerdo".

Finalmente, en lo que a la dimensión política se refiere, se debe notar que 14 de los sujetos que representan el $37 \%$ de los encuestados seleccionaron la categoría de respuesta positiva "De acuerdo", 11 de los sujetos que representan el 30\% de los encuestados seleccionaron la categoría de respuesta "Muy de acuerdo", 6 de los sujetos que representan 15\% de los sujetos encuestados seleccionaron la categoría de respuesta negativa "En desacuerdo", 4 de los sujetos que representan el 11\% de los encuestados 
seleccionaron la categoría de respuesta "Muy en desacuerdo", y 3 de los sujetos que representan el 7\% seleccionaron la categoría de respuesta "Ni de acuerdo, ni en desacuerdo".

La evidencia presentada en la tabla 1 permite inferir que, al caracterizar las dimensiones del desarrollo endógeno en la gerencia de las universidades del distrito de Santa Marta, el promedio de los resultados refleja que 11 de los sujetos que representan el 30\% de los encuestados y 10 de los sujetos que representan el $27 \%$ de los encuestados seleccionaron las categorías de respuestas positivas "De acuerdo" y "Muy de acuerdo" respectivamente. Mientras que 8 de los sujetos que representan el 20\% de los encuestados y 6 de los sujetos que representan el $16 \%$ de los encuestados seleccionaron las categorías de respuestas negativas "En desacuerdo" y "Muy en desacuerdo" respectivamente.

Los resultados reflejados en el gráfico 1 permiten inferir que, al sumar las categorías de respuestas positivas: 27 de los sujetos que representan el 73\% de los encuestados caracterizan la dimensión cultural del desarrollo endógeno en la gerencia, 25 de los sujetos que representan el $67 \%$ de los encuestados la dimensión organizacional, 25 sujetos que representan el 67\% de los encuestados la dimensión política, 24 de los sujetos que representan el $64 \%$ de los encuestados la dimensión tecnológica, 20 sujetos que representan el 54\% de los encuestados la dimensión ética, 18 de los sujetos que representan el $49 \%$ de los encuestados la dimensión social, y solo 10 de los sujetos que representan el $27 \%$ de los encuestados la dimensión económica.

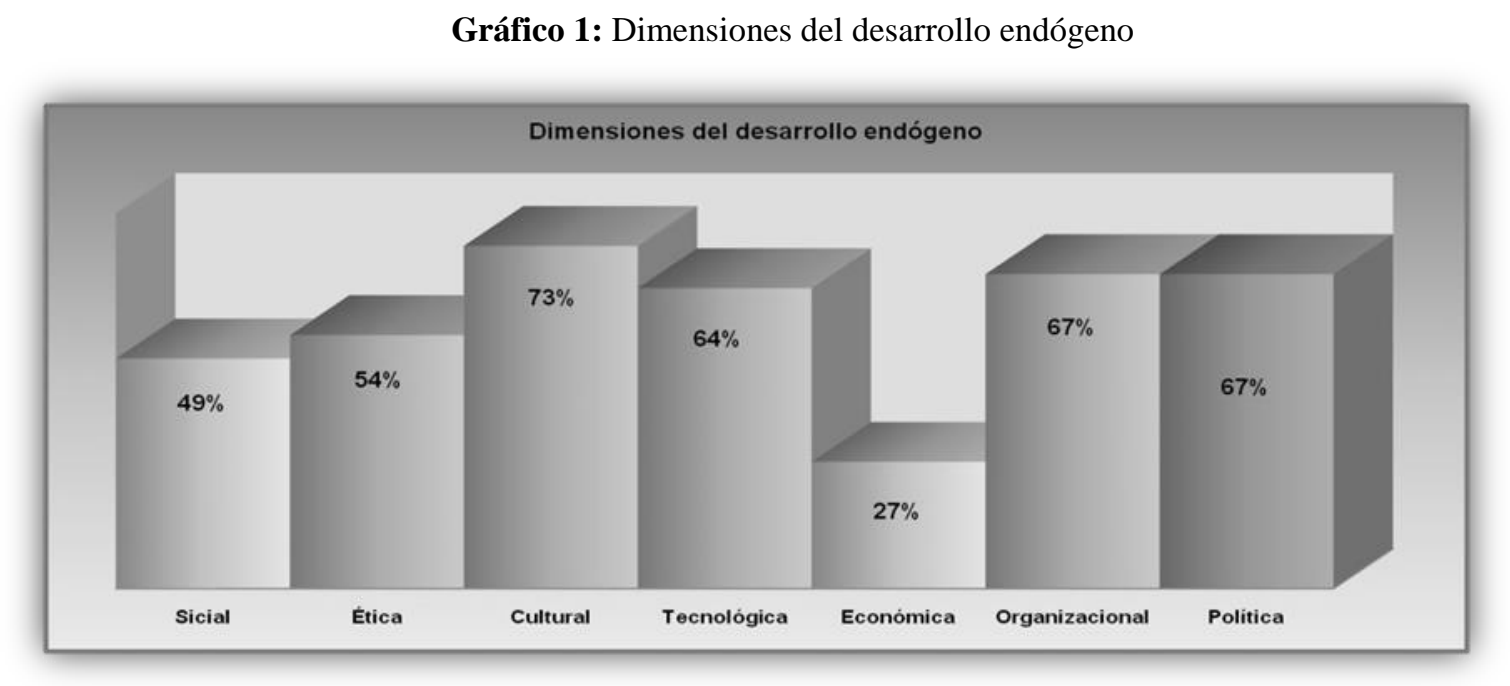

Nota: Elaboración propia (2016)

Asimismo, los resultados reflejados en el gráfico 1, para caracterizar las dimensiones del desarrollo endógeno en la gerencia de las universidades del distrito de Santa Marta, permiten hacer mención de lo indicado por Mas (2005), quien se refiere a la dimensión social acotando que el desarrollo endógeno es en sociedad, sus logros y propósitos, así como los recursos con los que cuenta, tienen por escenario y 
teología, lo social.

\section{5.- Discusión}

En un sentido particular, los procesos de desarrollo están condicionados por factores sociales como el espíritu de trabajo, la capacidad y las reglas que regulan las relaciones entre las personas y las organizaciones del territorio. Así, cuando en una organización se premia el esfuerzo y la ética de trabajo, la capacidad emprendedora es un valor social reconocido, y la movilidad social se estimula, la población está en capacidad de responder a los retos y desafíos, de manera creativa, considera Vásquez (2007).

En cuanto a la dimensión ética, Mas (2005) afirma que, todos los actos humanos requieren una valoración previa, esta valoración se realiza en atención a unos valores, los cuales, constituyen la esfera moral y cómo esta afecta poderosamente las elecciones y decisiones de las personas ejercen una gran influencia en todos los campos de la vida humana.

Asimismo, el autor señala que la dimensión cultural es asumida como la forma de vida de las personas, y que cada comunidad, país o nación, presenta una tipología de costumbres propias y singulares, así como creencias y tradiciones, formas de vida, de interacción y de acción, que le otorgan especial particularidad a un conjunto humano y social, siendo la cultura unos de los elementos claves del ámbito de sustentabilidad para el desarrollo endógeno.

Haciendo referencia sobre la dimensión tecnológica, Mas (2005) enuncia que la tecnología es una palanca clave para el desempeño de los procesos productivos, para el ejercicio organizacional y para la economía en general. Por otro lado, Alburquerque (2004) afirma que, la dimensión tecnológica del desarrollo endógeno implica mantener y dinamizar el potencial de creatividad, innovación y solución de los problemas.

En cuanto a la dimensión económica, el autor antes mencionado expone que en la economía se presenta la alternativa para la selección, producción y consumo de medios que otorgan satisfacción a los deseos humanos con base en las infinitas interacciones sociales y sobre la selección de estos medios se fundamenta la acción económica.

Por su parte, Vásquez (2007), considera que la dimensión económica está referida a la capacidad que demuestran las empresas endógenas para organizar los factores productivos con niveles de productividad suficientes para ser competitivos en los mercados, y caracterizada por: una elevada división del trabajo entre las empresas del sistema productivo local, una marcada especialización productiva, una multiplicidad de sujetos económico locales, una fácil difusión de información técnica y comercial mutua, un sistema de formación profesional construido por la propia localidad y, una fuerte integración entre las instituciones y la economía local. 
En el mismo sentido, Mas (2005) fundamenta la dimensión organizacional y gerencial indicando que el fenómeno del desarrollo endógeno asigna o impone como elemento clave de sustentación, la existencia de organizaciones eficientes que se dediquen a la producción de bienes y servicios. Además, se requiere la inclusión de otras organizaciones a los fines, de dar apalancamiento al sistema productivo general y a la comunidad social interesada en el desarrollo endógeno.

De igual manera el autor señala que, en la dimensión política del desarrollo endógeno, se pretende trazar los roles, concepciones, y estilos de comportamientos de los entes gubernamentales para apoyar y consolidar la estrategia de desarrollo endógeno. Esto significa que la política institucional de participación, representada por las forma de organización un conjunto de políticas territoriales permiten la creación de un entorno económico local favorable, capaz de desplegar las potencialidades locales y de proteger al territorio de interferencias externas, señala Vásquez (2007).

Sobre la base de estas referencias bibliográficas se debe apreciar que, la dimensión social del desarrollo endógeno está presente en la mayoría de los encuestados con respuestas positivas lo que significa que para la gerencia de las universidades en estudio es importante el espíritu de trabajo, para ello propicia capacidades emprendedoras en los empleados y estimula la población con capacidad de retos creativos.

Del mismo modo, la gerencia estimula los valores morales, respeta los campos de la vida para una convivencia en sociedad y considera que la moral de las personas se desempeña trascendentalmente en el desarrollo endógeno lo que evidencia la presencia de las características de la dimensión ética.

Los resultados arrojaron un alto porcentaje sobre la dimensión cultural, por lo que se concluye que la gerencia considera las costumbres que se engendran en el seno de una sociedad, fomenta el conocimiento científico entre los empleados y respeta las creencias de los empleados.

De igual forma, se evidencia la dimensión tecnológica por lo que se concluye que, la gerencia considera que la tecnología es clave para los procesos productivos, busca estrategias tecnológicas para promover el Desarrollo Endógeno y considera la necesidad de apropiarse de la tecnología.

En relación a la dimensión económica, los resultados reflejados en la investigación permiten evidenciar un porcentaje muy bajo sobre la presencia de sus características, en este sentido se concluye la que la gerencia de las universidades en estudio no estimula los medios que otorgan satisfacción a los deseos humanos, no considera los sujetos locales para el desarrollo endógeno y no busca la difusión de información académica.

Sobre las características de la dimensión organizacional y gerencial, se concluye que la gerencia impulsa el desarrollo endógeno en los empleados de la organización, ayuda a la generación de servicios 
para la región y contribuye con la comunidad social interesada en el desarrollo endógeno.

Las características de la dimensión política del desarrollo endógeno se hacen presente en los resultados que arrojaron, que para una mayoría significativa de los sujetos encuestados, la gerencia en las universidades en estudio propone pautas para consolidar la estrategia de desarrollo endógeno, permite la creación de un entorno económico local favorable y busca desplegar las potencialidades locales para proteger al territorio de interferencias externas.

En este sentido se infiere que, el desarrollo endógeno es la fuerza espiritual en la gerencia de las universidades para realizar un proceso de crecimiento económico y cambio estructural, que tiene como propósito orientara una comunidad hacia el logro de una mejor condición de vida. Asimismo, la gerencia ejecuta un conjunto de acciones o actividades seleccionadas e impulsadas por la totalidad de los miembros de una comunidad para alcanzar el futuro, entendiendo por comunidad una familia, un municipio, una ciudad, una región, un país, una nación o un grupo de países.

\section{6.- Conclusiones}

Las características de las dimensiones del desarrollo endógeno presentes en la gerencia de las universidades en estudio fueron las dimensiones: ética, cultural, tecnológica, organizacional y gerencial, y política; mientras que están ausentes las características de las dimensiones social y económica.

Estos resultados permitieron concluir que la gerencia de las universidades en estudio, considera las costumbres que se engendran en el seno de una sociedad, además, que la tecnología es clave para los procesos productivos, estimula los valores morales, ayuda a la generación de servicios para la región y propone pautas para consolidar la estrategia de desarrollo endógeno.

Se recomienda difundir y aplicar conocimiento sobre la importancia de las dimensiones del desarrollo endógeno reforzando la ética como la filosofía de la moral en el desarrollo endógeno, pues determina el marco valorativo social-individual de donde se propone el futuro y se preparan las condiciones necesarias para su consecución definitiva. Asimismo, la necesidad de organizar los factores productivos con niveles de productividad suficientes para ser competitivos en los mercados, difundiendo el trabajo entre las empresas del sistema productivo local, una marcada especialización productiva, una multiplicidad de sujetos económico locales, una fácil transmisión de información técnica y comercial mutua, un sistema de formación profesional construido por la propia localidad.

\section{7.- Referencias}

Alburquerque, F. (2004). Desarrollo económico local y descentralización en América Latina. Revista de la Cepal. $N^{\circ}$ 84. Instituto de Economía y Geografía, Consejo Superior de Investigaciones Científicas. España: Ministerio de Ciencia y Tecnología. 
Botello, E. (2013). Ética en la universidad. Madrid, España: Editorial Cyan.

Durán, S., Fuenmayor, A., Cárdenas, S. \& Hernández, R. (2016). Emprendimiento como proceso de responsabilidad social en instituciones de educación superior en Colombia y Venezuela. En Desarrollo Gerencial. Universidad Simón Bolívar-Colombia, 8(2), 58 - 75

Garizabal, M., Londoño, L. \& Uribe, A. (2015). Responsabilidad social en la universidad de la costa: visión, estrategia compartida. En Desarrollo Gerencial. Universidad Simón Bolívar 7(1), 175-190.

Gibson, I. (2006). Organizaciones, comportamiento, estructura, procesos. México: Editorial McGrawHill.

Kreitner, R. y Kinicki, A. (2003). Comportamiento organizacional: Conceptos, Problemas y Prácticas. México: Editorial Mc Graw Hill Interamericana.

Mas, M. (2005). Desarrollo Endógeno. Caracas, Venezuela: Editorial Panapo.

Mas, M. (2006). Desarrollo endógeno, Cooperación y Competencia. Caracas, Venezuela: Editorial Panapo.

Mas, M. (2008). Desarrollo endógeno y educación: estrategia de transformación. Edición ilustrada. Caracas, Venezuela: Editorial Panapo.

Morán, J. L. (2007). La Economía Comunal. Edición electrónica gratuita. Texto completo en http://www.eumed.net/libros-gratis/2007c/335/index.htm

Paz, A., Sánchez, J., Magdaniel, Y. \& Robles, C. (2017). Estrategias de la responsabilidad social como acción voluntaria en la Universidad de La Guajira. En Desarrollo Gerencial. Universidad Simón Bolívar-Colombia, 9(1), 126-143.

Tamayo y Tamayo, M. (2004). El Proceso de la Investigación Científica. 4ta Edición. México: Editorial: Limusa S.A.

Vásquez, A. (2007). Desarrollo endógeno. Teorías y políticas de desarrollo territorial. Red de Revistas Científicas de América Latina y el Caribe, España y Portugal. Sistema de Información Científica. Investigaciones Regionales, núm. 11, pp. 183-210. Madrid, España: Asociación Española de Ciencia Regional.

Zevallos, E. (2001). Experiencias Internacionales sobre las políticas para la empresa media. Santiago de Chile, Chile: Editorial McGrawHill. 